\title{
RHIC Au Beam in Run 2014
}

\author{
S.Y. Zhang
}

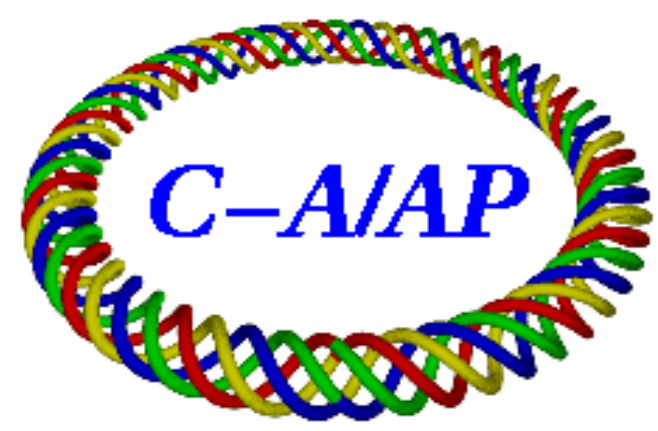

\section{Collider-Accelerator Department Brookhaven National Laboratory Upton, NY 11973}

\section{U.S. Department of Energy Office of Science, Office of Nuclear Physics}

Notice: This document has been authorized by employees of Brookhaven Science Associates, LLC under Contract No. DE-AC02-98CH10886 with the U.S. Department of Energy. The United States Government retains a nonexclusive, paid-up, irrevocable, world-wide license to publish or reproduce the published form of this document, or allow others to do so, for United States Government purposes. 


\title{
RHIC Au Beam in Run 2014
}

\author{
S.Y. Zhang \\ September 15, 2014
}

\begin{abstract}
$\mathrm{Au}$ beam at the RHIC ramp in run 2014 is reviewed together with the run 2011 and run 2012. Observed bunch length and longitudinal emittance are compared with the IBS simulations. The IBS growth rate of the longitudinal emittance in run 2014 is similar to run 2011, and both are larger than run 2012. This is explained by the large transverse emittance at high intensity observed in run 2012, but not in run 2014. The big improvement of the AGS ramping in run 2014 might be related to this change. The importance of the injector intensity improvement in run 2014 is emphasized, which gives rise to the initial luminosity improvement of $50 \%$ in run 2014, compared with the previous $\mathrm{Au}-\mathrm{Au}$ run 2011. In addition, a modified IBS model, which is calibrated using the RHIC Au runs from $9.8 \mathrm{GeV} / \mathrm{n}$ to 100 $\mathrm{GeV} / \mathrm{n}$, is presented and used in the study.
\end{abstract}

\section{RHIC Au beam in run 2014}

The observed Au bunch length and the calculated longitudinal emittance are studied for run 2011, run 2012, and run 2014, where the longitudinal emittance growth in ramp is dominated by the IBS (intra-beam scattering).

A model of IBS effect on RHIC Au beams is shown in [1]. Since the longitudinal emittance growth also depends on other beam parameters, such as the transverse emittance and momentum spread, the IBS studies also provide estimate of these parameters, which are more difficult in direct measurement.

In this study, a slightly modified IBS model from the one used in [1] is applied. The IBS rise time is calibrated according to RHIC Au runs at $9.8 \mathrm{GeV} / \mathrm{n}, 13.5 \mathrm{GeV} / \mathrm{n}, 19.5 \mathrm{GeV} / \mathrm{n}, 31.5 \mathrm{GeV} / \mathrm{n}$, and $100 \mathrm{GeV} / \mathrm{n}$. See details in the Section 2.

In Figure 1, typical Au beam bunch length evolution in ramp for $100 \mathrm{GeV} / \mathrm{n} \mathrm{Au}-\mathrm{Au}$ run 2011, $\mathrm{Cu}-\mathrm{Au}$ run 2012, and $\mathrm{Au}-\mathrm{Au}$ run 2014 is presented. The calculated longitudinal emittance in ramp is also presented. The simulated counterparts of the bunch length and longitudinal emittance are compared with the observed ones.

In Figure 2, the transverse emittance and the beam momentum spread shown in the IBS simulation are presented. To match the observed longitudinal eminence growth rate, much larger transverse emittance in run 2012 is used, which seems agreeable with the observed transverse emittance dependence on intensity in run 2012 [2]. 

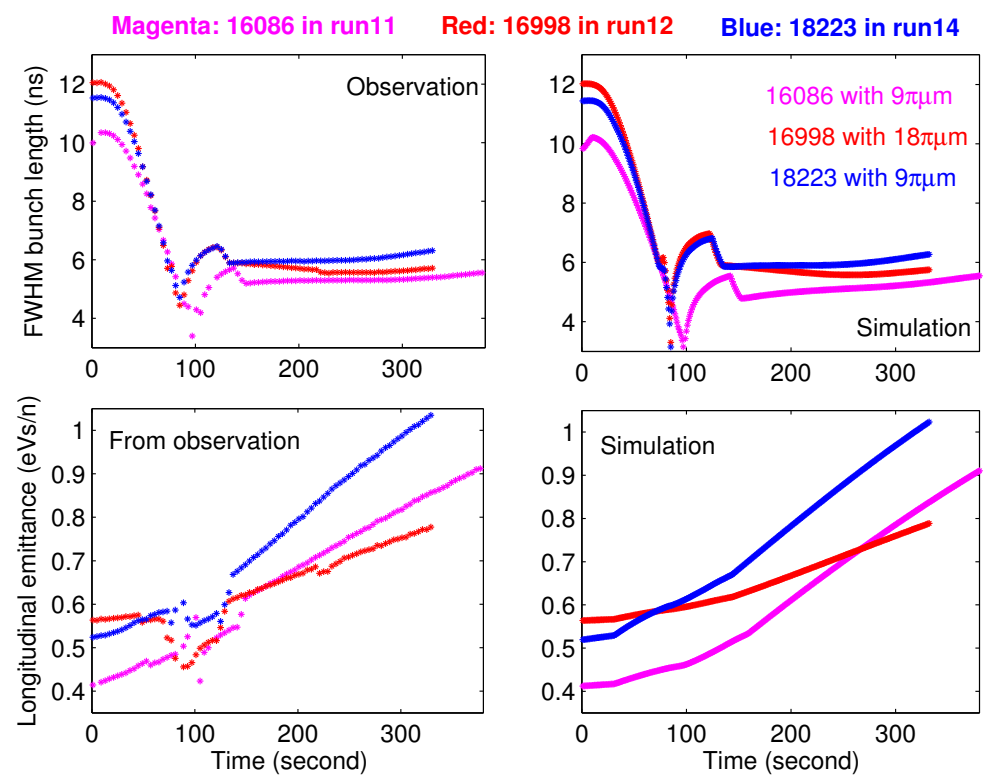

Figure 1: Typical Au beam bunch length evolutions in ramp of $100 \mathrm{GeV} / \mathrm{n} \mathrm{Au}-\mathrm{Au}$ run 2011, Cu-Au run 2012, and Au-Au run 2014 are shows. The corresponding longitudinal emittance is calculated from the lattice, rf, and Lorentz parameters. Simulation using the IBS model is compared with the observations.
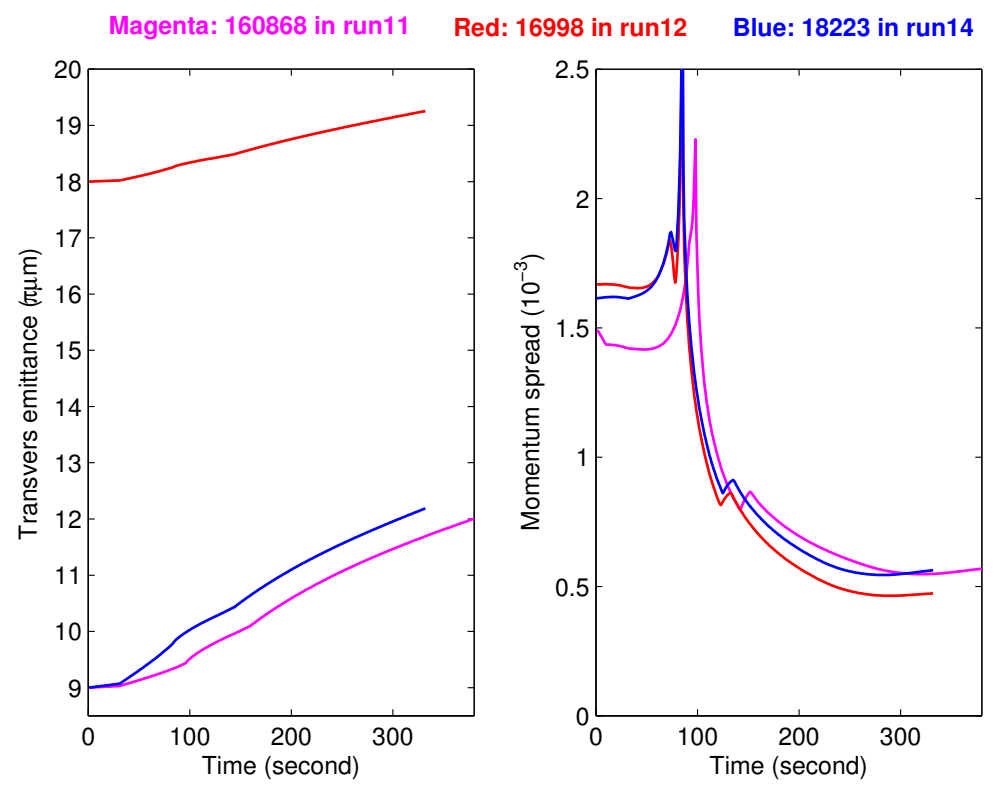

Figure 2: The IBS simulation for Au beam in ramp is also involved with the transverse emittance and the beam momentum spread, which are more difficult in direct observations. Note that the transverse emittance in run 2012 is much larger than run 2011 and run 2014, which is from the dependence on intensity observed in run 2012. 
A summary of the Au beam parameters in run 2014, compared with run 2011 and run 2012, is shown in Table 1.

\begin{tabular}{ccccc}
\hline \hline Fill & & 16086 & 16998 & 18223 \\
& & Run11 & Run12 & Run14 \\
\hline Ion source & & Tandem & EBIS & EBIS \\
Ramp lattice & & Au104 & CuAu12 & Au14 \\
Ramp time, $t$ & $s$ & 380 & 330 & 330 \\
RF Voltage at flatop, $V$ & $k V$ & 306 & 253 & 300 \\
Bunch intensity, $N_{b h}$ & $10^{9}$ & 1.50 & 1.51 & 1.69 \\
Initial longitudinal emittance, $\epsilon_{\ell}$ & $e V s / n$ & 0.41 & 0.56 & 0.52 \\
End longitudinal emittance, $\epsilon_{\ell}$ & $e V s / n$ & 0.91 & 0.78 & 1.02 \\
Initial transverse emittance, $\epsilon_{N}$ & $\pi \mu m$ & 9.0 & 18.0 & 9.0 \\
End transverse emittance, $\epsilon_{N}$ & $\pi \mu m$ & 12.0 & 19.3 & 12.2 \\
\hline \hline
\end{tabular}

Table 1. RHIC Au beam longitudinal and transverse emittance in ramp.

Comments are as follows:

1. In runs 2012 and 2014, EBIS is used as the ion source, and 4 Booster loads are merged into 1 bunch in the AGS. Initial longitudinal emittance at the RHIC injection is larger than run 2011, where the ion source is the Tandem.

2. Also in runs 2012 and 2014, the nominal lattice is used to replace the IBS suppression lattice used in run 2011. The dynamic aperture limit is improved, but regarding the IBS growth rate in RHIC ramp, the effect of the lattice change is limited.

3. Also in runs 2012 and 2014, the ramp time is cut from 380 second to 330 second, which reduced overall IBS growth on the ramp, for both longitudinal and transverse emittance.

4. The rf voltage used in runs 2011, 2012, and 2014 are not the same, but comparable. In later run 2014, the rf voltage is raised from $300 \mathrm{kV}$ to $400 \mathrm{kV}$. In this report, the early golden fill 18223 with $300 \mathrm{kV}$ rf voltage is used for the study.

5. In run 2012, a strong dependence of the transverse emittance on intensity is observed, which is reported in [2]. As the result, at high intensity, the transverse emittance at the RHIC injection is almost doubled from the typical $9 \pi \mu m$ in previous runs. This dependence is also presented for $\mathrm{Cu}$ and $\mathrm{U}$ beams in run 2012.

6. With the much larger transverse emittance in run 2012, the longitudinal emittance IBS growth in ramp is significantly reduced, which is consistent with the IBS simulation using large transverse emittance.

7. In run 2014, the transverse emittance dependence on intensity is not presented at the injector. The initial transverse emittance at the RHIC injection seems around $9 \pi \mu m$ again, similar to run 2011. 

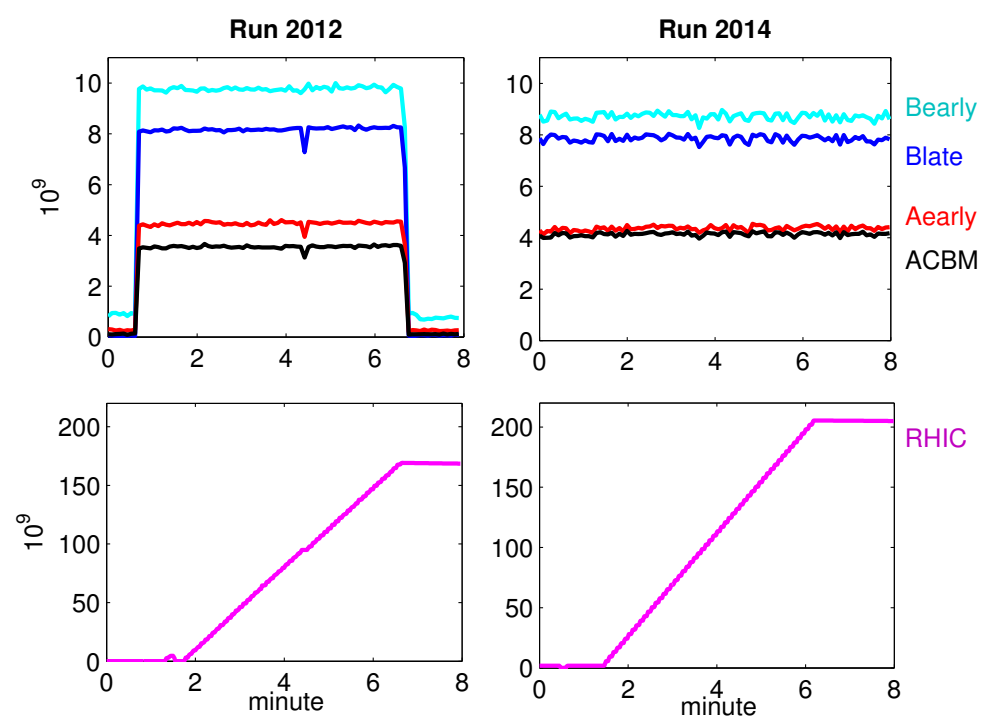

Figure 3: The Au beam transmission of run 2012 and 2014 in the injector is shown. The AGS transmission efficiency in run 2014 is significantly improved. As the result, the RHIC bunch intensity in run 2014 is improved by $20 \%$.

8. A possible reason of the change of run 2014 from run 2012 may be related to the significant improvement of the AGS transmission efficiency. In run 2012, the AGS efficiency is typically at $79 \%$, but in run 2014, it is improved to about $95 \%$. These are shown in Figure 3, and Table 2.

9. With the comparable Booster late intensity, the AGS CBM and the RHIC bunch intensity in run 2014 are both increased $20 \%$ from run 2012. This implies more than $40 \%$ improvement in RHIC initial luminosity.

\begin{tabular}{cccc}
\hline \hline Fill & & 16998 & 18371 \\
& & Run12 & Run14 \\
\hline Booster early intensity & $10^{9}$ & $9.7 / 2$ & $8.8 / 2$ \\
Booster late intensity & $10^{9}$ & $8.1 / 2$ & $8.0 / 2$ \\
AGS early intensity & $10^{9}$ & $4.5 / 2$ & $4.4 / 2$ \\
Strippping efficiency & $\%$ & 55 & 55 \\
AGS CBM intensity & $10^{9}$ & $3.5 / 2$ & $4.2 / 2$ \\
AGS efficiency & $\%$ & 78 & 95 \\
RHIC bunch intensity & $10^{9}$ & 1.52 & 1.85 \\
\hline \hline
\end{tabular}

Table 2. AGS transmission efficiency in run 2014 is significantly improved, which helped for high RHIC intensity, and may be also related to the better transverse emittance at RHIC.

The best Booster late intensity in run 2014 is comparable with that in run 2012. The stripping efficiency, which is dominant in the transmission from Booster late to AGS early, is both 55\% in 
run 2012 and run 2014. The big improvement of the AGS ramping gives rise to $20 \%$ increase of both the AGS CBM and the RHIC bunch intensity in run 2014, from run 2012.

To compare the $100 \mathrm{GeV} / \mathrm{n}$ Au-Au run 2014 with the previous Au-Au run 2011, Golden fills are presented in Table 3. Starting from Booster late intensity to RHIC collision, the intensity improvement is all about $20 \%$, which leads to the initial luminosity improvement of about $50 \%$. The transverse emittance is calculated from STAR ZDC, without correction. The cross section of $10 b$ is used.

It is clear that the RHIC bunch intensity improvement is still the key to luminosity improvement.

\begin{tabular}{ccccc}
\hline \hline Fill & & 16086 & 18371 & Increase of 18371 \\
& & Run11 & Run14 & $\%$ \\
\hline Booster late intensity & $10^{9}$ & 3.3 & 4.0 & 21 \\
AGS CBM intensity & $10^{9}$ & 1.75 & 2.10 & 20 \\
RHIC bunch intensity, injection & $10^{9}$ & 1.53 & 1.85 & 21 \\
AtR efficiency & $\%$ & 87 & 88 & 1 \\
RHIC bunch intensity, collision & $10^{9}$ & 1.33 & 1.63 & 22 \\
STAR ZDC & $k H z$ & 71 & 107 & 51 \\
Transverse emittance & $\pi \mu m$ & 10.6 & 10.7 & 1 \\
\hline \hline
\end{tabular}

Table 3. Comparison of $100 \mathrm{GeV} / \mathrm{n}$ Au-Au run 2011 and run 2014. All intensities from the injector to RHIC collision are improved by about $20 \%$ in run 2014 from run 2011, which give rise to the initial luminosity improvement of $50 \%$.

\section{Energy scaler modification of the IBS model}

It is known that the IBS rise time has a dependence on beam energy [3-5]. In RHIC, several low energy $\mathrm{Au}$ runs have been performed in past years, therefore, the observed longitudinal emittance growth at different energy might be used to calibrate the IBS rise time.

In Figure 4, the observed FWHM bunch length in 30 minutes with RHIC runs in different energy is presented. The IBS model of [1] is applied, which only has a dependence on the usual Lorentz parameters.

A systematic deviation of the simulated bunch length growth from the observed ones is shown in RHIC experiments. The deviation has a dependence on the energy, which indicates that additional energy scaler on the IBS model is needed.

In general, below the transition energy, which is approximately at $\gamma$ of 26.4 for IBS suppression lattice and 23.4 for the nominal lattice, the IBS model in [1] is over estimated, and at higher energy, the model is under estimated.

The detailed beam parameters of RHIC runs shown in Figure 4 are presented in Table 4. 

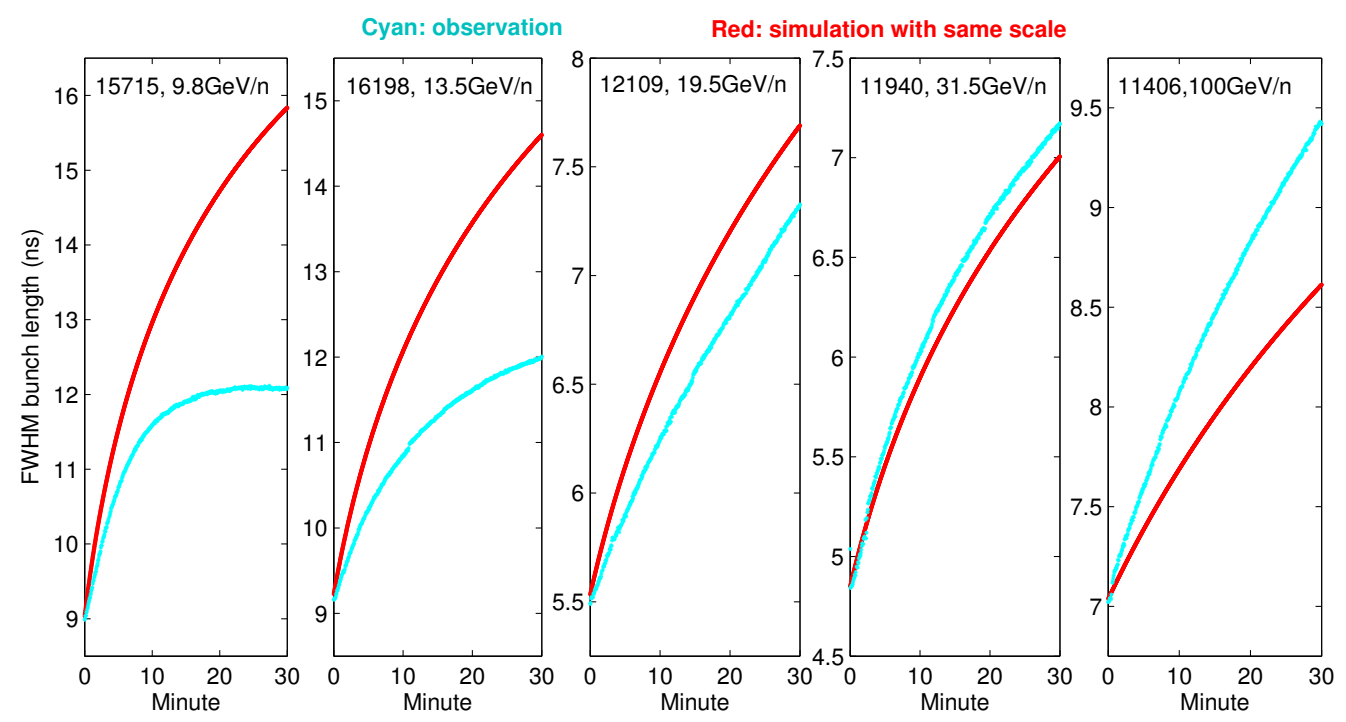

Figure 4: Typical Au bunch length growth in RHIC runs at $9.8 \mathrm{GeV} / \mathrm{n}, 13.5 \mathrm{GeV} / \mathrm{n}, 19.5 \mathrm{GeV} / \mathrm{n}$, $31.5 \mathrm{GeV} / \mathrm{n}$, and $100 \mathrm{GeV} / \mathrm{n}$ are shown. The IBS model with the same scale is used to simulate. A systematic deviation of the simulated growth from the observation is presented.
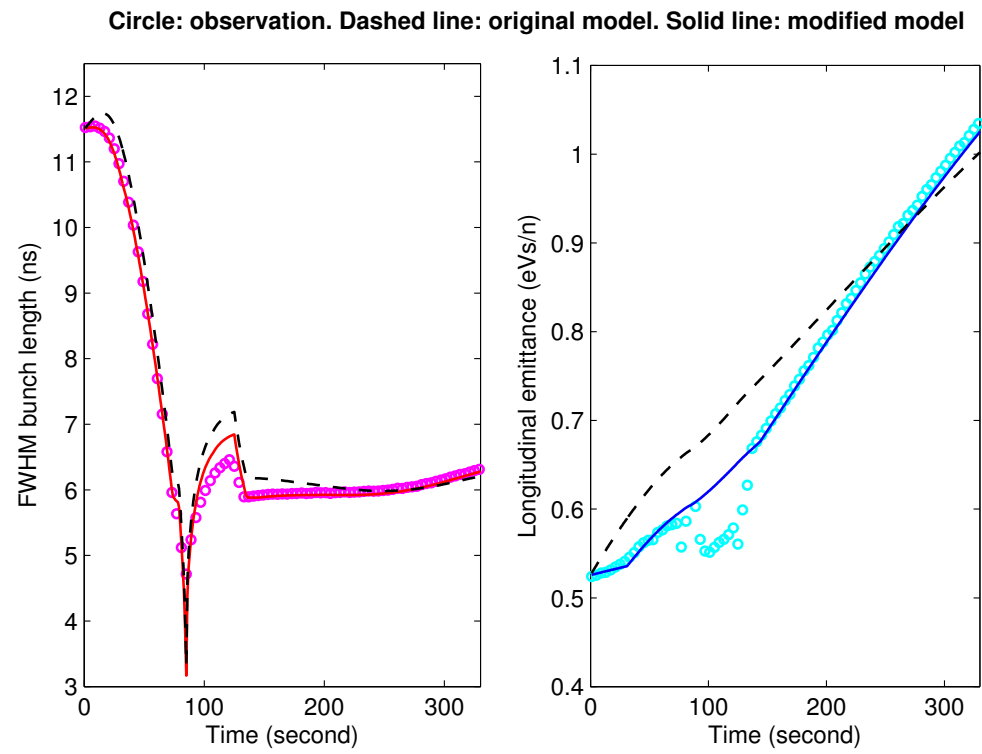

Figure 5: Modified IBS model is compared with the original one for the bunch length and longitudinal emittance growth in ramp of 18223. The overall growth is similar, but the modified model (solid line) lowered the growth rate below the transition, and raised it at the higher energy. 


\begin{tabular}{ccccccc}
\hline \hline Fill & & 15715 & 16198 & 12109 & 11940 & 11406 \\
\hline Beam energy & $G e V / n$ & 9.8 & 13.5 & 19.5 & 31.5 & 100 \\
Gamma, $\gamma$ & & 10.52 & 14.51 & 20.95 & 33.81 & 107.4 \\
Slippage, $\eta$ & $10^{-3}$ & -7.59 & -3.30 & -0.83 & 0.57 & 1.36 \\
Bunch intensity, $N_{b h}$ & $10^{9}$ & 0.88 & 1.52 & 0.82 & 0.96 & 0.96 \\
FWHM bunch length, $\tau_{F W H M}$ & $n s$ & 9.03 & 9.23 & 5.53 & 4.85 & 7.04 \\
Longitudinal emittance, $\epsilon_{\ell}$ & $e V s / n$ & 0.27 & 0.50 & 0.43 & 0.51 & 1.24 \\
Momentum spread, $d p / p$ & $10^{-3}$ & 1.15 & 1.49 & 1.35 & 1.42 & 1.85 \\
\hline \hline
\end{tabular}

Table 4. Beam parameters of RHIC Au runs with different energy.

In the study of the EBIS Au beam at RHIC ramp in 2012 [2], the overall IBS growth of the longitudinal and transverse emittance was the study goal, therefore, the simple model in [1], which is without energy scaler calibration, is used.

With a possible one more bunch merge at the AGS, the RHIC injection time would be doubled, it is of interest to use more accurate model.

The energy scalers of the IBS rise time is applied to that below the transition with $\gamma<25$, and with $\gamma<50$ but lager than the transition, and between 50 to 107, where the latter is the top energy of the RHIC Au ramp.

The modified model is applied to Fill 18223 in run 2014 with the observed FWHM bunch length and the longitudinal emittance in ramp. Figure 5 shows that the modified model has similar overall IBS growth in ramp with the original one, but the detailed match is better.

\section{References}

[1] S.Y. Zhang, "A Luminosity Model for RHIC Au Runs", C-A/AP/438, 11/1/2011

[2] S.Y. Zhang, "EBIS Au Beam at RHIC" , C-A/AP/459, 9/4/2012

[3] A. Sorenson, CERN-87-10, p.135, 1987

[4] A. Piwinski, Int. Conf. High Energy, Stanford, 1974

[5] A.V. Fedotov, "Comments on Simplified Treatment of Intrabeam Scattering", C-A/AP/168, $9 / 1 / 2004$ 